\title{
Collective Spontaneous Emission from Pairs of Quantum Dots: Long-Range vs. Short-Range Couplings
}

\author{
W. Abdussalam and P. Machnikowski* \\ Institute of Physics, Wrocław University of Technology, Wybrzeże Wyspiańskiego 27, 50-370 Wrocław, Poland

\begin{abstract}
We study the spontaneous emission from a coherently delocalized exciton state in a double quantum dot as a function of the distance between the dots, focusing on the similarities and differences between the cases of radiative (long-range, dipole) and tunnel coupling between the excitons in the dots. We show that there may be no qualitative difference between the collective emission induced by these two coupling types in spite of their essentially different physical properties.
\end{abstract}

PACS: 78.67.Hc, 42.50.Ct, 03.65.Yz

\section{Introduction}

Excitons delocalized in closely spaced quantum dots (QDs) recombine in a different way than in a single QD $[1,2]$. This effect is at least partly due to collective interaction of the two emitters with the quantum radiation field [3]. For non-interacting dots this collective effect appears only if the interband transition energies in the two QDs differ by no more than the emission line width, which requires the dots to be nearly identical, beyond the current technological possibilities. However, coupling between the dots restores the collective nature of the emission and leads to accelerated or slowed down emission even for dots with different transitions energies, which is manifested in the optical response from these systems $[4,5]$.

The two major couplings that may appear in a system of QDs are due to the Coulomb interactions and carrier tunneling. The former results from the coupling between the interband dipole moments associated with the excitons in the dots (sometimes referred to as the dispersion force) [6]. It has a long-range nature, with the typical $1 / R^{3}$ behavior at short distances (actually, this singularity is removed for charges distributed in a finite volume [7]) and an oscillating tail with an envelope decaying as $1 / R$ at distances larger than the resonant wave length. The tunnel coupling is a short-range interaction, which vanishes exponentially at distances on the order of a few nanometers.

In this contribution, we study the spontaneous emission from an exciton confined in a double quantum dot, focusing on the similarities and differences between the cases of radiative (long-range, dipole) and tunnel coupling between the excitons in the dots. We show that

* corresponding author; e-mail: Pawel.Machnikowski@pwr.wroc.pl for strictly identical dots the oscillating nature of the dipole coupling on long distances leads to non-monotonic dependence of the radiative decay rate on the inter-dot separation. However, for a double dot system with a realistic, technologically feasible mismatch of transition energies, the collective effects disappear completely well before these oscillations become relevant. In both cases, there is no qualitative difference between the emission induced by the long-range dipole interaction and that due to short-range tunnel couplings with appropriately chosen (but realistic) parameters, in spite of their essentially different physical origin and properties.

The paper is organized as follows. In Sect. 2, we describe the model of the system. Next, in Sect. 3, we present and discuss the results of numerical simulations. Finally, Sect. 4 concludes the paper.

\section{Model}

We consider two QDs placed in the $x y$ plane and shifted by a vector $\boldsymbol{r}_{12}$. Each QD is modeled as a two-level system (empty dot and one exciton). The Hilbert space of the double-dot system in our model is then spanned by the empty dot state $|00\rangle$, the two single exciton states $|10\rangle,|01\rangle$ corresponding to the exciton in the first and second dot, respectively, and the "molecular biexciton" state $|11\rangle$. The transition energies for the interband transitions in the two dots are

$$
E_{1}=\bar{E}+\epsilon, \quad E_{2}=\bar{E}-\epsilon .
$$

The dots are coupled by an interaction $V$ which can be either of dipole-dipole character (long-range dispersion force) or result from carrier tunneling (short-range, exponentially decaying interaction). We introduce the transition ("exciton annihilation") operators for the two dots, $\sigma_{1}=|00\rangle\langle 10|+| 01\rangle\left\langle 11\left|, \sigma_{2}=\right| 00\right\rangle\langle 01|+| 10\rangle\langle 11|$ and the exciton number operators $\hat{n}_{\alpha}=\sigma_{\alpha}^{\dagger} \sigma_{\alpha}, \alpha=1,2$. Using 
these operators, the Hamiltonian of the double-dot system is written in the frame rotating with the frequency $\bar{E} / \hbar$ in the form

$$
H_{0}=\epsilon\left(\hat{n}_{1}-\hat{n}_{2}\right)+V\left(\sigma_{1}^{\dagger} \sigma_{2}+\sigma_{2}^{\dagger} \sigma_{1}\right)+E_{\mathrm{B}} \hat{n}_{1} \hat{n}_{2},
$$

where the last term represents the biexciton shift.

The long-range dipole coupling is described by

$$
V=V_{\mathrm{lr}}=-\hbar \Gamma_{0} G\left(k_{0} r_{12}\right),
$$

where

$$
\Gamma_{0}=\frac{\left|d_{0}\right|^{2} k_{0}^{3}}{3 \pi \varepsilon_{0} \varepsilon_{\mathrm{r}}}
$$

is the spontaneous emission (radiative recombination) rate for a single dot, $\varepsilon_{0}$ is the vacuum permittivity, $\varepsilon_{\mathrm{r}}$ is the relative dielectric constant of the semiconductor, and

$$
k_{0}=\frac{n \bar{E}}{\hbar c},
$$

where $c$ is the speed of light and $n=\sqrt{\varepsilon_{\mathrm{r}}}$ is the refractive index of the semiconductor, and

$$
\begin{aligned}
& G(x)=\frac{3}{4}\left[-\left(1-\left|\hat{\boldsymbol{d}} \cdot \hat{\boldsymbol{r}}_{12}\right|^{2}\right) \frac{\cos x}{x}+\left(1-3\left|\hat{\boldsymbol{d}} \cdot \hat{\boldsymbol{r}}_{12}\right|^{2}\right)\right. \\
& \left.\quad \times\left(\frac{\sin x}{x^{2}}+\frac{\cos x}{x^{3}}\right)\right],
\end{aligned}
$$

where $\hat{\boldsymbol{r}}_{12}=\boldsymbol{r}_{12} / r_{12}$ and $\hat{\boldsymbol{d}}=\boldsymbol{d} / d$, where $\boldsymbol{d}$ is the interband matrix element of the dipole moment operator which is assumed identical for both dots. For a heavy hole exciton, $\boldsymbol{d}=\left(d_{0} / \sqrt{2}\right)[1, \pm \mathrm{i}, 0]^{\mathrm{T}}$, so that for a vector $\boldsymbol{r}_{12}$ in the $x y$ plane one has $\left|\hat{\boldsymbol{d}} \cdot \hat{\boldsymbol{r}}_{12}\right|^{2}=1 / 2$. The tunnel coupling is described by

$$
V=V_{\text {sr }}=V_{0} \mathrm{e}^{-r_{12} / r_{0}} \text {. }
$$

The effect of the coupling to the radiation field is accounted for by including the dissipative term in the evolution equations, which describes radiative recombination of excitons. The equation of evolution of the density matrix is then given by [8]:

$$
\dot{\rho}=-\frac{\mathrm{i}}{\hbar}\left[H_{0}, \rho\right]+\sum_{\alpha, \beta=1}^{2} \Gamma_{\alpha \beta}\left[\sigma_{\alpha} \rho \sigma_{\beta}^{\dagger}-\frac{1}{2}\left\{\sigma_{\alpha}^{\dagger} \sigma_{\beta}, \rho\right\}_{+}\right],
$$

where $\Gamma_{11}=\Gamma_{22}=\Gamma_{0}, \Gamma_{12}=\Gamma_{21}=\Gamma_{0} F\left(k_{0} r_{\alpha \beta}\right)$, with

$$
\begin{aligned}
& F(x)=\frac{3}{2}\left[\left(1-\left|\hat{\boldsymbol{d}} \cdot \hat{\boldsymbol{r}}_{12}\right|^{2}\right) \frac{\sin x}{x}+\left(1-3\left|\hat{\boldsymbol{d}} \cdot \hat{\boldsymbol{r}}_{12}\right|^{2}\right)\right. \\
& \left.\quad \times\left(\frac{\cos x}{x^{2}}-\frac{\sin x}{x^{3}}\right)\right],
\end{aligned}
$$

and $\{\ldots, \ldots\}_{+}$denotes the anticommutator. The diagonal decay rates $\Gamma_{\alpha \alpha}$ describe the emission properties from a single dot, while the off-diagonal terms $\Gamma_{\alpha \beta}, \alpha \neq \beta$, account for the interference of emission amplitudes resulting from the interaction with a common reservoir and are responsible for the collective effects in the emission.
In our simulations, we use the parameters for a typical InAs/GaAs QD system: $\Gamma_{0}=1 \mathrm{~ns}^{-1}, n=3.3, \bar{E}=$ $1.3 \mathrm{eV}$. For the tunnel coupling we choose the amplitude $V_{0}=2.19 \mathrm{meV}$ and the range $r_{0}=2.03 \mathrm{~nm}$, which makes the values for the tunnel and dipole couplings similar for inter-dot distances around $6 \mathrm{~nm}$.
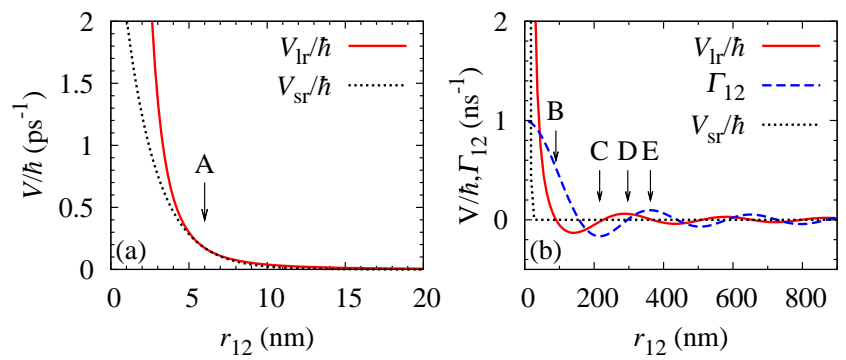

Fig. 1. The interference term of the decay rate $\Gamma_{12}$ and the short- and long-range coupling amplitudes $V_{\mathrm{lr}}, V_{\mathrm{sr}}$ as a function of the inter-dot distance. In (a), the small distance section is shown, while in (b) the oscillating tail at larger distances is visible. Let us note the different scales in (a) and (b).

The values of the two couplings as well as the interference term of the decay rate $\Gamma_{12}$ are plotted as a function of the distance between the dots in Fig. 1. In this figure we mark the distance values for which the decay will be discussed in the next section.

\section{Results}

In Fig. 2 we show the results of the numerical simulations based on Eq. (1). In each plot, the average number of excitons in the system is shown as a function of time for identical $\operatorname{dots}(\epsilon=0)$ and for slightly non-identical dots with $\epsilon=0.01 \mathrm{meV}$. The initial state in all the cases is chosen to be $(|01\rangle+|10\rangle) / \sqrt{2}$. We study the decay of exciton population for various distances between the dots and compare the evolution for the two kinds of couplings.

For identical dots, the exciton decay time for the delocalized initial state strongly depends on the distance between the dots. This is due to the oscillations and decay of the interference term $\Gamma_{12}$. For dots placed at a short distance (case $A$ ), $\Gamma_{12} \sim \Gamma_{0}$ and the decay has a strongly collective character, which is manifested by the faster emission visible in Fig. 2a,b [3]. The collective effect gets weaker as the distance between the dots grows and $\Gamma_{12}$ decreases $(B)$. For some values of the distance, $\Gamma_{12}<0(C)$. Then, the amplitudes for photon emission from the two dots interfere destructively and the decay gets slower than the usual exponential decay with the rate $\Gamma_{0}$ (the initial state becomes subradiant). Whenever $\Gamma_{12}=0$, the decay rate is the same as for an individual dot $(D)$. Comparison of Fig. 2a and b shows that for identical dots, these effects do not depend on the coupling and are therefore the same, irrespective of the presence and physical nature of the interaction between the dots. 


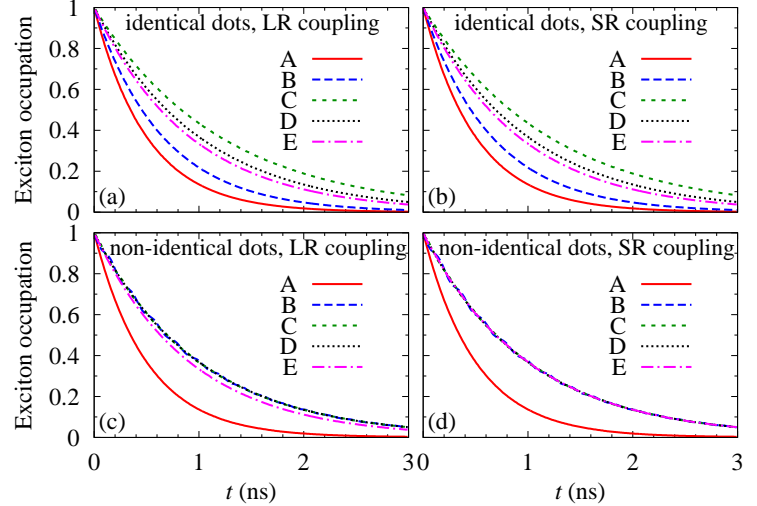

Fig. 2. The exciton occupation (the average number of excitons in the system) for an initial single-exciton state corresponding to a coherently delocalized superposition. Parts (a) and (b) show the evolution for a pair of identical dots coupled by long-range dipole forces and by short-range tunnel coupling, respectively. Parts (c) and (d) show the evolution for a pair of non-identical dots, for the two kinds of couplings as previously. The labels $A, \ldots, E$ refer to the values of the inter-dot distance marked in Fig. 1.

For dots that differ by the relatively small transition energy mismatch of $2 \epsilon=0.02 \mathrm{meV}$, almost this whole non-monotonic dependence of the emission rate on the distance disappears. The reason is that the oscillations of the interference term take place in the distance range where the coupling between the dots is very weak and is dominated already by a small energy mismatch assumed here, which destroys collectivity of the emission process [3]. The only exception is the smallest distance shown in this plot, where the coupling is sufficiently strong. By comparing Fig. 2c and d one can see that also in this case, the evolution of the exciton occupation is nearly the same for both systems. Here the tunnel coupling parameters have been deliberately chosen to assure the same coupling strength around the $6 \mathrm{~nm}$ distance. At larger distances both couplings are negligible compared to the energy mismatch.

\section{Conclusions}

We have shown that the radiative decay of exciton occupation in a pair of coupled quantum dots depends on the distance between the dots as a result of the spatial dependence of the interference term governing the interaction with the quantum electromagnetic field. For non-identical dots, the emission rate depends on the interplay of the energy mismatch between the dots and the coupling between them. Although the two kinds of couplings that are present in the system (tunneling and dipole interaction) have essentially different physical nature and properties, they may lead to the same dynamics of the observed collective emission.

We believe that these findings may shed some light on the interpretation of the experiment [9] in which enhanced emission was observed in a quantum dot ensemble in which the dipole coupling energies on the typical inter-dot distances were much smaller than the average transition energy mismatch between the dots. Indeed, as we have shown, the tunnel coupling leads to the same effect as the long-range dipole interaction but it can be stronger than the latter at short distances. Hence, it seems very likely that short-range tunnel coupling between some pairs of dots can be responsible for the observed collective emission effect in QD ensembles.

\section{Acknowledgments}

This work was supported by the Foundation for Polish Science under the TEAM programme, co-financed by the European Regional Development Fund.

\section{References}

[1] C. Bardot, M. Schwab, M. Bayer, S. Fafard, Z. Wasilewski, P. Hawrylak, Phys. Rev. B 72, 035314 (2005).

[2] P. Borri, W. Langbein, U. Woggon, M. Schwab, M. Bayer, S. Fafard, Z. Wasilewski, P. Hawrylak, Phys. Rev. Lett. 91, 267401 (2003).

[3] A. Sitek, P. Machnikowski, Phys. Rev. B 75, 035328 (2007).

[4] A. Sitek, P. Machnikowski, Phys. Rev. B 80, 115319 (2009).

[5] A. Sitek, P. Machnikowski, Phys. Rev. B 80, 115301 (2009).

[6] M.J. Stephen, J. Chem. Phys. 40, 669 (1964).

[7] P. Machnikowski, E. Rozbicki, Phys. Status Solidi B 246, 320 (2009).

[8] R.H. Lehmberg, Phys. Rev. A 2, 883 (1970).

[9] M. Scheibner, T. Schmidt, L. Worschech, A. Forchel, G. Bacher, T. Passow, D. Hommel, Nature Phys. 3, 106 (2007). 